\title{
036 ASSOCIATION OF APATHY WITH FRONTAL LOBE DYSFUNCTION IN AMNESTIC MILD COGNITIVE IMPAIRMENT AND ALZHEIMER'S DISEASE
}

doi:10.1136/jnnp-2013-306103.36

Manoj George, Tim Whitfield, Zuzanna Walker

Objective The primary aim of this study is to examine an association between apathy and frontal lobe dysfunction in patients with memory problems. We also aimed to look into the association between apathy and praxis.

Method This was a retrospective cross sectional study. We selected 160 consecutive patients diagnosed with Alzheimer's dementia and Amnestic Mild Cognitive Impairment who had a comprehensive battery of neuropsychological tests and a behaviour rating scale of interest for this study recorded in the database. Correlation between apathy with and without depression were tested against frontal lobe test including Trail making A, Trial making B, Letter Fluency, Ideational Fluency, Category fluency, Abstract Thinking and Executive functioning subtest of CAMGOG-R.

Results Statistically significant relationship were found between apathy and executive function scores, ideational fluency scores, abstract thinking and category fluency scores.

Conclusion Apathy is negatively related to executive function and ideational fluency. This finding has important clinical significance because poor scores in executive function influence memory abilities by preventing people to employ compensatory strategies that can help them remember information and maintain functional abilities. It is also associated with greater neuropsychiatric disturbances especially a greater degree of agitated and disinhibited behaviour. 